\title{
RELCASI
}

\section{Participación de los Usuarios en la Evaluación de la Calidad de los Sistemas de Información}

\author{
Juan M. Gómez Reynoso \\ Universidad Autónoma de Aguascalientes, jmgr@correo.uaa.mx \\ Mónica R. Brizuela Sandoval \\ Universidad Autónoma de Aguascalientes, mbrizuel@correo.uaa.mx
}

Follow this and additional works at: https://aisel.aisnet.org/relcasi

\section{Recommended Citation}

Gómez Reynoso, Juan M. and Brizuela Sandoval, Mónica R. (2009) "Participación de los Usuarios en la Evaluación de la Calidad de los Sistemas de Información," RELCASI: Vol. 2 : Iss. 1 , Paper 1.

DOI: $10.17705 / 1$ relc.00006

Available at: https://aisel.aisnet.org/relcasi/vol2/iss1/2

This material is brought to you by the AIS Journals at AIS Electronic Library (AISeL). It has been accepted for inclusion in RELCASI by an authorized administrator of AIS Electronic Library (AISeL). For more information, please contact elibrary@aisnet.org. 


\section{R E L C A S I $\quad 02 \quad 01 \quad 2009$}

\section{REVISIA HAINOAMERICANA}

Y DEL CARIBE DE LA

\section{ASOCIACIÓN DE SISTEMAS

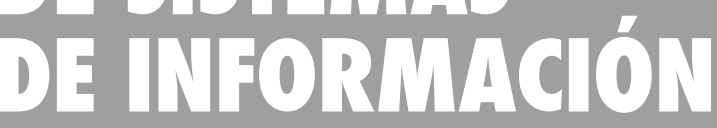

11 - Participación de los Usuarios en la Evaluación de la Calidad de los Sistemas de Información Juan Manuel Gómez Reynoso y Mónica Brizuela Sandoval

35- Impacto del Adiestramiento, Habilidades en Tecnología de la Información y Gerencia de Proyectos en el Éxito de Implementaciones de Sistemas Integrados ERP Miguel Maldonado y Martin Santana

$55 \cdot$ Distribución Geográfica de los Estudiantes de Ingeniería del ITAM en la Ciudad de México:

Caso de Estudio Marcelo Meija y Quauhti Martinez 


\section{$\begin{array}{llllllllll}R & E & L & C & A & S & \mid & 02 & 01 & 2009\end{array}$}

\section{REVISTA LATLNOAMERICANA}

ASOCAACÓ Y DE CARTB DE LA DE SISIEMAS DE INFORMAción

Editor:

Carlos Ferran 
Editor:

Carlos Ferran

Penn State Great Valley Pennsylvania State University 30 E Swedesford Road Malvern, PA 19355 U.S.A. cferran@psu.edu

Comité Editorial:

Carlos Dorantes Tecnológico de Monterrey, México cdorante@itesm.mx

Carlos Ferran Pennsylvania State University,

USA cferran@psu.edu

Martha Garcia-Murillo Syracuse University, USA mgarciam@syr.edu

David Montesinos INCAE, Costa Rica

David.Montesinos@incae.edu

Carlos J. Navarrete California State Polytechnic University, USA cjnavarrete@csupomona.edu

James B. Pick University of Redlands, USA James_Pick@redlands.edu

Guillermo Rodríguez-Abitia Universidad Nacional Autonoma de México grdrz@unam.mx

Volumen 2 Número 1, 2009 Portada: Maria Elena Repiso C) 2009 RELCASI ISSN 1937-8823

(en línea) ISSN 1937-8831 www.relcasi.org

\section{Revista Latinoamericana} y del Caribe de la Asociación de Sistemas de Información RELCASI

\section{TABLA DE CONTENIDO}

Volumen 2 Número 1, 2009

NOTA EDITORIAL

PARTICIPACIÓN DE LOS USUARIOS EN LA EVALUACIÓN DE LA CALIDAD DE LOS SISTEMAS DE INFORMACIÓN

Juan Manuel Gómez Reynoso y

Mónica Brizuela Sandoval

IMPACTO DEL ADIESTRAMIENTO, HABILIDADES EN TECNOLOGÍA DE LA INFORMACIÓN Y GERENCIA DE PROYECTOS EN EL ÉXITO DE IMPLEMENTACIONES DE SISTEMAS INTEGRADOS ERP

Miguel Maldonado y

Martín Santana

DISTRIBUCIÓN GEOGRÁFICA DE LOS ESTUDIANTES DE INGENIERIA DEL ITAM EN LA CIUDAD DE MÉXICO: CASO DE ESTUDIO

Marcelo Mejía y

Quauhtli Martinez

POLÍTICA EDITORIAL 
Editor-in-Chief:

Carlos Ferran

Penn State Great Valley Pennsylvania State University 30 E Swedesford Road Malvern, PA 19355 U.S.A. cferran@psu.edu

Editorial Board:

Carlos Dorantes Tecnológico de Monterrey, México cdorante@itesm.mx

Carlos Ferran Pennsylvania State University, USA cferran@psu.edu

Martha Garcia-Murillo Syracuse University, USA mgarciam@syr.edu

David Montesinos INCAE, Costa Rica David.Montesinos@incae.edu

Carlos J. Navarrete California State Polytechnic University, USA cjnavarrete@csupomona.edu

James B. Pick University of Redlands, USA James Pick@redlands.edu

Guillermo Rodríguez-Abitia Universidad Nacional Autonoma de México grdrz@unam.mx

Volume 2 Number 1, 2009 (C) 2009 RELCASI Cover: Maria Elena Repiso ISSN 1937-8823 (on-line) ISSN 1937-8831 www.relcasi.org

\section{Revista Latinoamericana y del Caribe de la Asociación de Sistemas de Información RELCASI}

TABLE OF CONTENT

Volume 2 Number 1, 2009

EDITORIAL

END-USER PARTICIPATION IN INFORMATION SYSTEMS QUALITYEVALUATION

Juan Manuel Gómez Reynoso y

Mónica Brizuela Sandoval

THE IMPACT OF TRAINING, IT SKILLS AND PROJECT MANAGEMENT ON ERP SYSTEMS IMPLEMENTATIONS SUCCESS

Miguel Maldonado y

Martín Santana

GEOGRAPHICAL DISTRIBUTION OF ITAM'S ENGINEERING STUDENTS IN MEXICO CITY: A CASE STUDY

55

Marcelo Mejía y

Quauhtli Martínez

EDITORIAL POLICY

74

CALL FOR ARTICLES 


\title{
Participación de los Usuarios en la Evaluación de la Calidad de los Sistemas de Información
}

\section{End-User Participation in Information Systems Quality Evaluation}

\author{
Juan Manuel Gómez Reynoso \\ Universidad Autónoma de Aguascalientes, jimgr@correo.uaa.mx \\ Mónica Brizuela Sandoval \\ Universidad Autónoma de Aguascalientes, mbrizuel@correo.uaa.mx
}

\begin{abstract}
RESUMEN
Los diseñadores de software siempre intentan realizar sistemas con calidad. Un problema es que no siempre disponen de técnicas/herramientas adecuadas. Muchos sistemas de información son desarrollados a la medida para problemas únicos en las organizaciones. La evaluación empírica por parte de los usuarios de al menos dos prototipos de software permite evaluar el mismo, reduciendo así, algunos de los problemas que se presentan en la etapa de implantación. Las mediciones se deben llevar a cabo en ambientes controlados y por usuarios que tienen un nivel de experiencia lo suficientemente adecuado para poder hacer dicha evaluación. Es por eso que surge la siguiente pregunta: ¿La evaluación de sistemas de información por parte de los usuarios incrementa su calidad? Evidencias encontradas en el presente estudio muestran que la respuesta a la pregunta es sí.
\end{abstract}

Palabras Claves: Sistemas de información, usabilidad, usuarios, evaluación empírica, calidad del software.

\section{ABSTRACT}

Software developers always try to creating high-quality information systems. One important issue is that not always have availability of proper techniques/tools. Many information systems are developed to address a special need within organizations. Empirical evaluation of at least two information system prototypes by end-users helps to reduce the number of issues that could be present during the implantation phase. IS quality measurement has to be conducted in a controlled environment and made by end-users that has enough experience to do such evaluation. Thus, the following question arises: "Evaluation by end-users improves IS quality?" Outcomes of our study suggest that end-user evaluation improves quality.

Keywords: Information systems, usability, end-users, empirical evaluation, software quality. 


\section{MARCO TEÓRICO}

Muchas de las organizaciones enfrentan la batalla diaria por mantenerse competitivas. Especialmente, en un mundo globalizado la competencia suele ser más intensa. La competencia que enfrentan las organizaciones les presenta retos provenientes de distintas fuentes tales como: Gobierno, clientes, proveedores, etc. Para ser competitivos, las organizaciones deben ser más eficientes en todos sus procesos, tanto productivos como administrativos. La eficiencia depende mucho de los empleados, pero también de las herramientas que a éstos se les provean, así como de los métodos y técnicas que están implementadas en la organización. Es importante recordar que las personas son el elemento más valioso de las organizaciones (Drucker, 1993, 1999; Miller, 1995) y también el más crítico para el éxito de las mismas. Un personal altamente capacitado y especializado puede ayudar considerablemente al éxito de la organización.

Mediante el uso de herramientas adecuadas y pertinentes las organizaciones pueden mejorar su eficiencia, de forma tal que pueden ser más competitivas en costos, acaparar un nicho de mercado, o ser identificados de entre todos sus competidores (Applegate, Austin, \& McFarlan, 2003). Una de dichas herramientas es el uso de sistemas de información (SI) como apoyo de sus procesos y en la toma de decisiones. Las tecnologías de información (TI) han proporcionado nuevas formas de ayudar a las organizaciones (Buch \& Bartley, 2002) mediante la construcción de nuevas herramientas y métodos (Kekäle, Pirolt, \& Falter, 2002). No todos los problemas de las organizaciones son de tipo universal por lo que un mismo SI no resuelve el mismo problema en dos o más organizaciones diferentes aunque el problema sea dentro del mismo contexto, la instanciación del mismo varía de una organización a otra. Entonces, la adaptación de los SI a la organización es clave para que los empleados optimicen los recursos de la misma.

El uso de SI es de gran utilidad para cualquier tipo de organización ya que les permite realizar una mejor toma de decisiones, simplificar sus actividades cotidianas, establecer alianzas, mejorar la imagen de la organización con sus clientes, entre otras. Es por eso que es necesario que muchos de los SI sean desarrollados a la medida de las necesidades particulares de la organización (Pressman, 2005; Sommerville, 2006), ya que los problemas que resuelven son únicos en las misma.

El desarrollo de software es una actividad que puede tener alto impacto en las organizaciones, ya que muchas de las actividades que se llevan a cabo son soportadas por software. Por ejemplo, nómina, contabilidad, inventarios, entre otras. Un sistema con calidad y libre de errores puede ayudar a la optimización de recursos, así como contribuir a la administración de la organización de manera eficiente. 
Existen distintas opciones para la adquisición de SI por parte de las organizaciones. Entre las más conocidas son: comprarlo ya hecho, desarrollados dentro de la organización, ser desarrollados por empresas especializadas, o que los datos sean procesados mediante outsourcing. Cada una de las opciones debe utilizarse tomando en cuenta aspectos relevantes del medio ambiente y del proyecto. Por ejemplo, Dennis et al. ( 2002) proporcionan una serie de sugerencias, las cuales se muestran en la Tabla 1.

Tabla 1. Comparación para selección entre estrategias de adquisición de software (A. Dennis, Wixom, B. H., \& Tegarden, D., 2002)

\begin{tabular}{|l|c|c|c|}
\hline & $\begin{array}{c}\text { Usar desarrollo por los } \\
\text { usuarios cuando... }\end{array}$ & $\begin{array}{c}\text { Compra el software ya } \\
\text { hecho cuando... }\end{array}$ & $\begin{array}{c}\text { Usar outsourcing } \\
\text { cuando... }\end{array}$ \\
\hline $\begin{array}{l}\text { Necesidad del } \\
\text { negocio }\end{array}$ & $\begin{array}{c}\text { La necesidad del } \\
\text { negocio es única } \\
\text { la empresa }\end{array}$ & $\begin{array}{c}\text { La necesidad del } \\
\text { negocio es común }\end{array}$ & $\begin{array}{c}\text { La necesidad del } \\
\text { negocio no es crítica } \\
\text { para la empresa }\end{array}$ \\
\hline $\begin{array}{l}\text { Habilidades para el } \\
\text { proyecto }\end{array}$ & $\begin{array}{c}\text { Existe experiencia } \\
\text { técnica y funcional } \\
\text { desarrollar habilidades } \\
\text { internas }\end{array}$ & $\begin{array}{c}\text { Existe experiencia } \\
\text { funcional }\end{array}$ & $\begin{array}{c}\text { No existe experiencia } \\
\text { técnica o funcional }\end{array}$ \\
\hline $\begin{array}{l}\text { Administración del } \\
\text { proyecto }\end{array}$ & $\begin{array}{c}\text { El proyecto cuenta con } \\
\text { un líder de proyecto } \\
\text { muy capaz asín como una } \\
\text { metodología probada }\end{array}$ & $\begin{array}{c}\text { El proyecto cuenta con } \\
\text { un líder de proyecto } \\
\text { que puede coordinar los } \\
\text { esfuerzos de los } \\
\text { vendedores }\end{array}$ & $\begin{array}{c}\text { El proyecto cuenta con } \\
\text { un líder de proyecto } \\
\text { muy capaz a nivel igual } \\
\text { que el alcance del trato } \\
\text { a outsource }\end{array}$ \\
\hline Tiempo necesario & $\begin{array}{c}\text { El tiempo de entrega es } \\
\text { flexible }\end{array}$ & $\begin{array}{c}\text { El tiempo de entrega es } \\
\text { corto }\end{array}$ & $\begin{array}{c}\text { El tiempo de entrega es } \\
\text { corto y flexible }\end{array}$ \\
\hline
\end{tabular}

Aun persiste el problema de desarrollar software de alta calidad a tiempo y dentro de presupuesto (Pressman, 2005). Tradicionalmente, el desarrollo de SI se ha llevado a cabo sin medir la calidad estimada antes de su implantación (Pressman, 2005; Sommerville, 2006). Los errores, requerimientos incompletos o mejoras son atendidos hasta que el usuario final trabaja con los SI. Esta forma de desarrollo de sistemas puede causar pérdidas económicas, de recursos, de clientes o, en algunos casos, tener un costo de oportunidad.

Frecuentemente, los desarrolladores de software tienden a ignorar al usuario final durante el desarrollo del SI que les es solicitado. Esto debido a que de manera inconsciente se ven afectados por los paradigmas de la ingeniería de software reportados en la literatura (Pressman, 2005; Sommerville, 2006). Tanto Pressman (2005) como Sommerville (2006) mencionan que, frecuentemente, el usuario es tomado en cuenta solamente durante las etapas de recolección de información e identificación de requerimientos $\mathrm{y}$, en ocasiones, los desarrolladores no validan los requerimientos con los usuarios.

Los usuarios tienen diferencias en muchos aspectos tanto demográficos como en preferencias de interacción. Esto impacta directamente en la forma en que prefieren interactuar con los SI. Por ejemplo, un usuario con 
mucha experiencia preferirá un diálogo iniciado por él con la computadora, al contrario de uno novato que prefiere que la computadora dirija el diálogo (Pressman, 2005; Sommerville, 2006). Entonces, el usuario asigna un nivel alto de importancia a la forma en que él puede utilizar el sistema. Esto es importante, ya que el usuario es quien realmente determina el éxito si utiliza y aprovecha al máximo el SI o fracaso, si no lo hace. Además, la mayoría de los SI utilizados en la organizaciones son comprados como paquetes estándar (por ejemplo, nómina, contabilidad, entre otros) ya que resulta más rápida su implantación así como de un costo menor a otras opciones y, generalmente, este software comprado no puede ser adaptado a las necesidades particulares de la organización (Sommerville, 2006) debido a que el proveedor sólo entrega el código ejecutable, más no el código fuente. Cabe mencionar, que las organizaciones frecuentemente recurren al desarrollo de SI por parte de empresas dedicadas a esta actividad y, en ocasiones, por personal propio. Asimismo, los errores en la programación o en el diseño del software no son detectados hasta que el sistema está siendo operado por los usuarios. Esto podría ocasionar la pérdida de información valiosa, difícil o imposible de recuperar. Para entonces, los costos asociados se han elevado mucho comparados con el costo de un SI libre de errores. Los costos se elevan debido a que es necesario rediseñar o darle mantenimiento correctivo a las aplicaciones, la recuperación y reparación de datos dañados, esto sin mencionar el costo de oportunidad que se podría presentar debido a la necesidad de suspender las actividades a las que el sistema está apoyando (Pressman, 2005; Sommerville, 2006). En conclusión, esto provoca muchos problemas y pérdidas a las organizaciones.

Existen múltiples metodologías para el desarrollo de SI. Dichas metodologías han sido descritas ampliamente en la literatura (A. Dennis, Wixom, \& Roth, 2006; A. Dennis, Wixom, B. H., \& Tegarden, D., 2002; Pressman, 2005; Satzinger, Jackson, \& Burd, 2009; Sommerville, 2006; E. Turban, McLean, \& Wetherbe, 2001). Estas varían en la forma de llevar a cabo cuatro etapas principales: planeación, análisis, diseño y codificación. Entre las metodologías más utilizadas tenemos las siguientes:

- Metodología estructurada. También llamado "modelo clásico", "modelo en cascada" o "modelo tradicional". Esta metodología es muy simple y se caracteriza por llevar a cabo las etapas en forma de una cascada, es decir, una etapa no puede iniciar sino hasta que la anterior haya sido concluida. En esta metodología el involucramiento de los usuarios se lleva acabo principalmente en la identificación de requerimientos, así como en la implantación.

- Metodología orientada a objetos es un enfoque de la ingeniería de software que modela un SI como un grupo de objetos que interactúan entre sí. Este enfoque representa un dominio en términos de conceptos 
compuestos por verbos y sustantivos, clasificados de acuerdo a su dependencia funcional. En este método de desarrollo de SI se crea un conjunto de modelos utilizando una simbología estándar. Esta metodología aplica técnicas de modelado de objetos para analizar los requerimientos para un contexto y para diseñar una solución para mejorar los procesos del negocio.

- La Metodología por prototipos se inicia con la definición de los objetivos para el SI. Posteriormente, se identifican los requisitos conocidos y las áreas de donde es necesaria más definición. Posterior a esto, se plantea un prototipo inicial y se presenta el modelo al usuario. El diseño inicial se centra en una representación de aquellos aspectos del software que serán visibles para el usuario final y conduce a la construcción de un prototipo, el cual es evaluado por el usuario para una retroalimentación. Con la retroalimentación, se refinan los requisitos del SI que se está desarrollando. La iteración ocurre cuando el prototipo se ajusta para satisfacer las necesidades del evaluador. Esto permite que al mismo tiempo el desarrollador entienda mejor lo que se debe hacer y el cliente puede ver resultados a corto plazo. Generando con esto, más confianza.

- Metodología en Espiral es un modelo evolutivo que conjuga la naturaleza iterativa del método por prototipos con los aspectos controlados y sistemáticos de la metodología en cascada. En el modelo en Espiral, el software se construye por medio de una serie de versiones incrementales. En las primeras iteraciones la versión incremental no necesariamente es un software, ya que podría ser un modelo en papel. En las iteraciones finales se desarrollan versiones cada vez más completas del SI objetivo. El modelo se divide en un número de Actividades de marco de trabajo, llamadas "regiones de tareas". En general existen entre tres y seis regiones de tareas aunque es importante mencionar que existen variantes del modelo.

Tomando en cuenta las distintas formas que existen para desarrollar SI, creemos que la evaluación de al menos dos prototipos del SI de forma empírica por parte de los usuarios permite identificar y resolver muchos de los problemas que pueden impedir que los usuarios finales exploten al máximo el SI. Además, la participación de los usuarios en las etapas tempranas podría ayudar a reducir el rechazo potencial que se tiene hacia un nuevo SI en la etapa de implantación.

Los profesionales de las áreas relacionadas con el desarrollo de software siempre tienen en mente que se deben desarrollar los SI con calidad. Un problema para realizar esto es el hecho de que no siempre tienen disponibles técnicas y/o herramientas que les permitan hacer la evaluación completamente y, en muchos casos, los conocimientos para hacerlo. Es por eso que surge la siguiente pregunta: ¿La evaluación de SI mediante 
usabilidad por parte de los usuarios ayuda a mejorar la calidad del software? Los resultados encontrados en el presente estudio nos permite afirmarlo.

Los usuarios interactúan con los SI a través de las interfaces. El estándar ISO 13407 define Usabilidad como: La usabilidad de una interfaz es la medida de la efectividad, eficiencia y satisfacción con la cual usuarios específicos pueden cumplir metas específicas en un ambiente particular con esa interfaz. Entonces, si un SI específico tiene usabilidad significa que los usuarios del mismo podrán realizar su trabajo de mejor manera.

De acuerdo a Nunamaker (1992) una forma de desarrollar SI es mediante prototipos. El prototipeo es un método que provee una versión del sistema con la que los usuarios pueden interactuar rápidamente de forma tal que se puedan refinar los requerimientos durante el desarrollo del SI (A. Dennis, Wixom, B. H., \& Tegarden, D., 2002). Investigaciones previas (E. Turban, 1995; Efraim Turban, Leidner, McLean, \& Wetherbe, 2006; E. Turban et al., 2001) mencionan que el número de prototipos necesarios a desarrollar hasta finalizar el sistema es desconocido. Adicionalmente, otros investigadores (J. G. Walls, Widmeyer, \& El Sawy, 1992; J.G. Walls, Widmeyer, \& El Sawy, 2004) proponen que los prototipos sean evaluados con el fin de medir si el actual prototipo es una mejora real del anterior, pero también puede evaluarse si tiene mejor usabilidad que el prototipo anterior.

Generalmente, la evaluación de los SI es llevada a cabo por parte de los desarrolladores de manera gentil (Pressman, 2005; Sommerville, 2006). Pero un desarrollador no actúa ni piensa como lo hace un usuario. Por lo tanto, proponemos que la evaluación se lleve a cabo iterativamente en ambientes estructurados por parte de los usuarios. Es decir, medir una primera vez de forma tal que se puede tener un valor base, así como detectar problemas; resolver los problemas detectados y evaluar nuevamente hasta tener la calidad deseada. Las mediciones se deben llevar a cabo en ambientes controlados y realizadas por usuarios que tienen un nivel de experiencia lo suficientemente adecuado para poder calificar el sistema desarrollado.

Tiwana y McLean (2005) argumentan que el desarrollo de sistemas es una actividad creativa que involucra expertise, ideas y habilidades de varios individuos. Una de las principales actividades en los departamentos de sistemas es el desarrollo y mantenimiento de software (Pressman, 2005; Sommerville, 2006), por lo que la creciente demanda para crear nuevos sistemas o modificar los existentes pone presión extra a los desarrolladores, lo cual podría provocar que los sistemas desarrollados no sean cuidadosamente evaluados y revisados. Consideramos que ningún usuario está dispuesto a usar software que tiene baja calidad, incompleto y/o con errores, ya que todo esto podría generar la pérdida y/o daño de la 
información existente $\mathrm{y}$, en consecuencia, pérdidas económicas $\mathrm{y}$ problemas mayores. Entonces, es muy importante que los sistemas sean desarrollados con calidad suficiente. Creemos que la calidad del software se puede mejorar mediante la evaluación por usuarios de manera empírica.

Zayaraz et al. (2005) argumentan que la calidad del software tiene diversos aspectos, pero la calidad general puede expresarse en términos de la combinación de varios de ellos. Amasaki et al. (2005) creen que un software con calidad ayuda a reducir los costos de mantenimiento, así como satisfacer los requerimientos de los usuarios, quienes son los que en realidad conocen perfectamente los requerimientos que cada sistema debe cumplir. Además, son los usuarios que tienen en mente la forma más adecuada para interactuar con el sistema de software.

La calidad del software se mejora reduciendo el número de fallas mediante pruebas exhaustivas (Amasaki et al., 2005) mientras que el tiempo y/o los recursos existentes lo permitan (Pressman, 2005; Sommerville, 2006). La mayoría de las fallas en los proyectos de software se deben a que los usuarios no son involucrados, existen requerimientos incompletos y deficiencias ingenieriles (Boehm, 2006). Entonces, si los usuarios evalúan los sistemas, estarán evaluando tanto la calidad como lo completo del sistema. Dicha evaluación debe llevarse a cabo de forma ordenada.

Generalmente, quienes realizan las pruebas del sistema son el grupo de desarrolladores, pero no lo evalúan de la forma en que un usuario lo operaría. Lo evalúan de la forma en que un experto lo usaría y, más aún, de forma inconsciente, lo operan como desarrollador más que como usuario final. Proponemos que los usuarios formen parte de los evaluadores del sistema, retroalimenten a los desarrolladores, éstos tomen la evaluación recibida $\mathrm{y}$, en base a ella, hagan los ajustes necesarios al sistema, todo en forma iterativa.

Si los SI son evaluados con el fin de garantizar la calidad por parte de los usuarios se pueden atacar varios problemas potenciales de adopción de SI: los usuarios se sienten ignorados por los desarrolladores; por ejemplo, los usuarios rechazan una nueva forma de trabajo. Consideramos que si los SI son evaluados durante la programación/pruebas por parte de los usuarios se podrían minimizar dichas barreras.

La intención que se tiene al desarrollar para usabilidad descansa en la meta de que la persona que use el producto lo haga rápido y fácilmente de forma tal que pueda cumplir sus tareas (Dumas \& Redish, 1999). La definición de Dumas se basa en cuatro puntos: 1) usabilidad significa enfocarse a los usuarios, 2) la gente usa los productos para el desempeño de actividades productivas, 3) los usuarios son gente ocupada que trata de cumplir tareas, y 4) los usuarios deciden cuando un producto es fácil de usar o no. Aunque las pruebas de usabilidad no sean físicamente peligrosas, tienen que ser realizadas de forma ética (Thompson, 2003). Las 
conclusiones de las pruebas de usabilidad tienen un gran impacto en el diseño de un sistema (Rutherford, 2000) puesto que toman en cuenta aspectos que son considerados como importantes por el usuario final del sistema. Los investigadores argumentan que las pruebas de usabilidad sólo son factibles durante el desarrollo del sistema (Mehlenbacher, 1993), lo cual permite ser aplicado cuando la metodología de desarrollo del software es por prototipeo.

"El software debería sufrir pruebas rigurosas, ser evaluado para investigar su funcionalidad, así como el análisis de usabilidad para examinar el diseño de interacción" (De Villiers, 2004, p. 284). La usabilidad mejora la calidad del producto (Rutherford, 2000), lo cual impacta en las potencialidades de un SI. Entonces, es sumamente importante probar la usabilidad (Thompson, 2003). Sin embargo, las pruebas de usabilidad no son muy utilizadas (Mehlenbacher, 1993) y el no usarlas podría disminuir la calidad de los productos. En consecuencia, sugerimos que los nuevos SI sean sometidos a pruebas de usabilidad, sobre todo aquellos que son diseñados para la gente que tiene poca o ninguna experiencia en la utilización de computadoras. La Figura 1 muestra el proceso seguido para la evaluación de SI sugerido por nosotros.

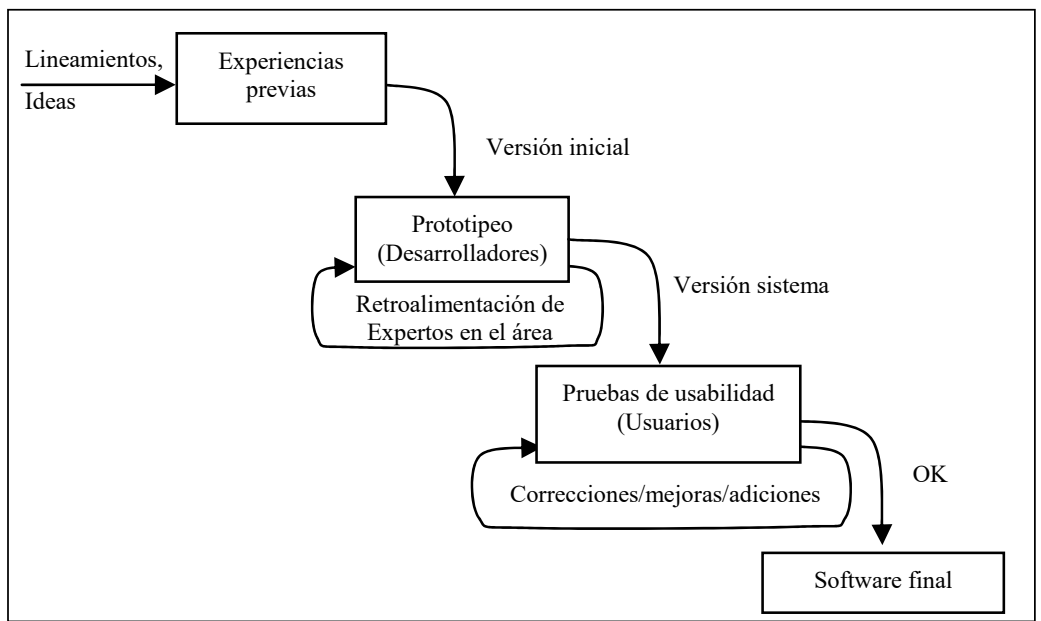

Figura 1. Proceso de desarrollo propuesto

Para satisfacer las demandas de programación se han desarrollado varias metodologías para el desarrollo de sistemas. Cada metodología indica un nivel diferente de participación del usuario en el desarrollo del proyecto. Algunas metodologías demandan que el usuario participe sólo al inicio (ingeniería de requerimientos) y al final (implantación del sistema) -i.e. desarrollo en cascada. Otras, que su participación sea más interactiva durante todo el ciclo de desarrollo (i.e. prototipeo); pero ninguna metodología demanda que el usuario evalúe de forma empírica cada versión que se está generando del sistema. 
Consideramos que la metodología por prototipos para desarrollo de sistemas de software es la mejor forma de aplicar la evaluación de calidad mediante usabilidad. Dicha metodología de forma natural requiere una participación activa por parte de los usuarios durante todo el ciclo de desarrollo, entonces, es factible que cuando se tenga un prototipo "final" se trabaje con el usuario en la evaluación que proponemos.

La siguiente figura muestra la forma en que se lleva a cabo el desarrollo de sistemas mediante la metodología de prototipeo, tomando como base la metodología propuesta por Dennis et. al (2002) e incluyendo nuestra propuesta, la cual se muestra en la parte inferior de la figura.

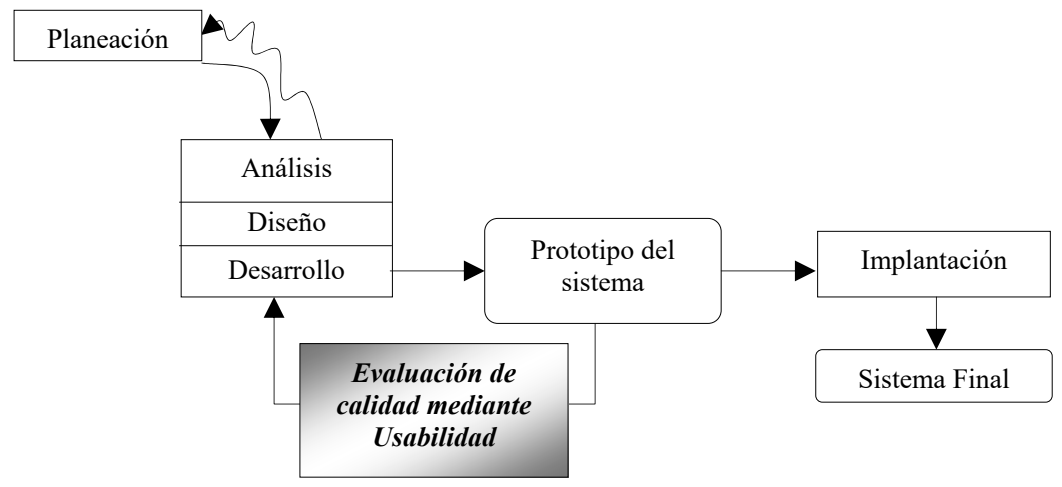

Figura 2. Metodología por prototipeo

La usabilidad ha estado presente como atributo de calidad desde el final de los 70s (Juristo \& Ferre, 2006) y está siendo reconocida ampliamente como un atributo el cual se debe tomar en cuenta al diseñar SI para que éstos tengan calidad (Bosch \& Juristo, 2003; Juristo \& Ferre, 2006). Además, usabilidad es una teoría que permite la evaluación de la calidad de un sistema de software (Dumas \& Redish, 1999; UW, 2004). Constantine y Dockwood (2003) mencionan que la ingeniería de software es frecuentemente débil cuando se trata de atender áreas críticas tales como requerimientos de usuario, usabilidad, interfaces de usuario y diseño de la interacción. Adicionalmente, ellos mencionan que estos asuntos han sido ya minimizados, ignorados o dejados de lado para que sean atendidos por otras disciplinas. Se propone probar que la medición de la usabilidad incrementa la calidad del software producido. La medición debe realizarse mediante el uso de estadística descriptiva y pruebas de hipótesis. De esta forma, se puede tener la medición de calidad del sistema desarrollado, así como la conclusión de las hipótesis planteadas.

Debido a todo lo anterior, pensamos que es importante el estudiar si la participación directa en la evaluación de desarrollo de SI tiene un efecto positivo en la calidad final del software. Por lo tanto, consideramos que 
"Un software desarrollado por medio de prototipos y evaluado con teorías de usabilidad durante su desarrollo tiene mejor calidad que un software no evaluado con teorías de usabilidad durante su desarrollo".

\section{METODOLOGÍA}

\section{Participantes}

Para probar si la participación de los usuarios en la evaluación de los SI mejora la calidad de los mismos se realizaron dos estudios con dos grupos cada uno. En cada estudió participó un grupo como desarrollador y un grupo como evaluador. Todos los grupos pertenecen a la carrera de Ingeniero en Sistemas Computacionales en una universidad pública en México, la cual está relacionada con el desarrollo, mantenimiento e implantación de SI. Los desarrolladores fueron los responsables de crear el sistema de información y los evaluadores tomaron el papel de usuario final de los sistemas desarrollados. A continuación se describen ambos estudios.

Estudio de grupos estrategia con usabilidad (GCU). Los participantes que tomaron el papel de desarrolladores de sistemas fueron 23 estudiantes cursando la materia de Ingeniería de Software en el noveno semestre. Adicionalmente, 22 estudiantes de un grupo diferente participaron como evaluadores de los sistemas desarrollados.

Estudio de grupos estrategia sin usabilidad (GNU). Participaron 18 estudiantes como desarrolladores cursando la materia de Ingeniería de Software en el noveno semestre, así como un grupo diferente de 20 estudiantes participaron como evaluadores de los sistemas desarrollados.

\section{Diseño de la Investigación}

El estudio GCU se condujo como se describe a continuación: los desarrolladores crearon un primer prototipo de un SI utilizando las especificaciones proporcionadas por los investigadores. El prototipo fue entregado a todos los evaluadores, los cuales lo ejecutaron. Posterior a la ejecución, respondieron una encuesta acerca de la usabilidad del prototipo. Los resultados obtenidos en la evaluación fueron entregados como retroalimentación a los desarrolladores. Con estos resultados, los desarrolladores realizaron correcciones, modificaciones y mejoras a sus sistemas, generando así el segundo prototipo. El segundo prototipo se entregó nuevamente a los evaluadores para realizar el mismo procedimiento. Los resultados de ambas evaluaciones se compararon con el fin de evaluar el nivel de mejora mostrado y verificar si existió una mejora significativa en la usabilidad del sistema.

El estudio GNU se condujo como se describe a continuación: los desarrolladores crearon un único prototipo de un SI utilizando las mismas especificaciones que al GCU proporcionadas por los investigadores. El prototipo fue entregado a todos los evaluadores, los cuales lo ejecutaron. 
Los resultados de la evaluación se analizaron con el fin de evaluar el nivel de usabilidad logrado.

Como se menciona previamente la medición se llevó a cabo usando una encuesta, la cual se evaluó mediante estadística descriptiva para cada muestra. La encuesta incluye dos apartados:

i) Preguntas para recabar información demográfica acerca del evaluador.

ii) Preguntas de opinión acerca del sistema.

a. Cerradas en una escala de 7 de Liekert para recabar aspectos de usabilidad acerca del software

b. Abiertas para recabar aspectos que permiten mejorar el software desarrollado

La encuesta fue elaborada tomado como componentes los sugeridos por McCall en su teoría de calidad de sistemas, así como el ISO 9126 (ISO, 1991). De manera general, el método desarrollado consistió en establecer, en base al análisis e integración de los modelos y estándares de calidad de ambos, qué factores de la calidad de un producto de software deben evaluarse desde el punto de vista del usuario final (tales como usabilidad, confiabilidad, etc.), cómo deben medirse $\mathrm{y}$ en qué términos. A continuación se describen brevemente los componentes de calidad de ambas sugerencias.

\section{Factores de Calidad de McCall}

McCall, Richards y Walters (1977) propusieron una clasificación de los factores que afectan la calidad del software. Dichos factores (ver Figura 3) se concentran en tres aspectos importantes de un producto de software: sus características operativas, su capacidad para experimentar cambios y su capacidad para adaptarse a nuevos entornos.

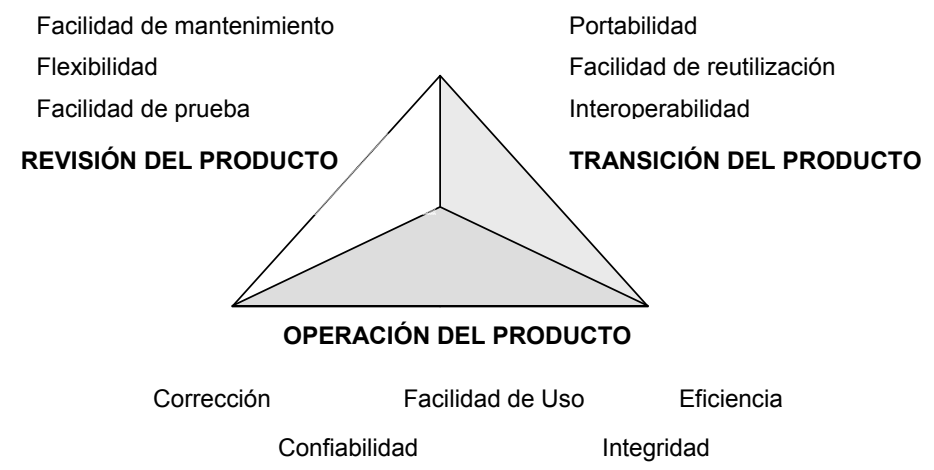

Figura 3. Factores de calidad del software de McCall (Adaptado de Sommerville, 2006)

Sommerville (2006) describe cada uno de los componentes del triángulo de McCall de la siguiente manera:

1. Revisión del producto, el cual se divide en: 
- Facilidad de Mantenimiento. Es el esfuerzo necesario para localizar y corregir un error en un programa.

- Flexibilidad. Es el esfuerzo necesario para modificar un programa en operación.

- Facilidad de Prueba. Es el esfuerzo que demanda probar un programa con el fin de asegurar que realiza su función

2. Transición del Producto, el cual se divide en:

- Portabilidad. Es el esfuerzo necesario para transferir el programa de un entorno de hardware o software a otro.

- Facilidad de Reutilización. Es el grado en que un programa (o partes de él) puede reutilizarse en otras aplicaciones (en relación con el empaquetamiento y alcance de las funciones que realiza el programa).

- Interoperabilidad. Es el esfuerzo necesario para acoplar un sistema con otro.

3. Operación del Producto, el cual se divide en:

- Corrección. Es el grado en que un programa cumple con su especificación y satisface los objetivos que propuso el cliente.

- Confiabilidad. Es el grado en que se esperaría que un programa desempeñe su función con la precisión requerida.

- Eficiencia. Es la cantidad de código y de recursos de cómputo necesarios para que un programa realice su función.

- Integridad. Es el grado de control sobre el acceso al software o los datos por partes de las personas no autorizadas.

- Facilidad de Uso. Es el esfuerzo necesario para aprender, operar y preparar los datos de entrada de un programa e interpretar la salida.

\section{Factores de Calidad del Estándar ISO 9126}

El ISO 9126 (ISO, 1991) es un estándar que se desarrolló como un intento por identificar los atributos de calidad para el software. Éste identifica seis atributos claves de la calidad, que se describen a continuación.

1. Funcionalidad. Es el grado en que el software satisface las necesidades que indican los siguientes sub-atributos: idoneidad, exactitud, interoperabilidad, cumplimiento y seguridad.

2. Confiabilidad. Es la cantidad de tiempo en que el software está disponible para usar según los siguientes sub-atributos: madurez, tolerancia a fallas y facilidad de recuperación.

3. Facilidad de uso. Es la facilidad con que se usa el software de acuerdo con los siguientes sub-atributos: facilidad de comprensión, facilidad de aprendizaje y operabilidad. 
4. Eficiencia. Es el grado en que el software emplea en forma óptima los recursos del sistema, como lo indican los siguientes sub-atributos: comportamiento en el tiempo, comportamiento de los recursos.

5. Facilidad de mantenimiento. Es la facilidad con que se repara el software de acuerdo con los siguientes sub-atributos: facilidad de análisis, facilidad de cambio, estabilidad y facilidad de prueba.

6. Portabilidad. Es la facilidad con que se lleva el software de un entorno a otro según los siguientes sub-atributos: adaptabilidad, facilidad para instalarse, cumplimiento, facilidad para reemplazarse.

A partir de los elementos identificados como factores en la evaluación de la calidad del producto de software, se creó una serie de preguntas para la evaluación de estos factores, así como la escala de respuestas posible para su valoración. Cada pregunta se respondió en una escala de 1 a 10 , donde a mayor valor, mejor calificación.

Este instrumento fue validado mediante una prueba piloto con 14 alumnos de la asignatura de Ingeniería de Software de la carrera de Ingeniería en Sistemas Computacionales de la Universidad Autónoma de Aguascalientes, México. Para tal efecto, los estudiantes evaluaron con el instrumento creado, una aplicación de software. Una vez aplicado el instrumento, se evaluaron los resultados obtenidos. Para la evaluación de los factores considerados mediante estadística descriptiva. Con los resultados de este estudio piloto, se rediseño el instrumento de medición.

Además, se llevó a cabo un análisis semántico de las preguntas abiertas de forma tal que se pudieron identificar tendencias, sugerencias, mejoras, errores, entre otros.

Posteriormente, se compararon los SI finales mediante pruebas $t$ con el fin de probar la hipótesis planteada.

\section{Parámetros de Medición}

Los parámetros de medición de la usabilidad que de forma preliminar se podrían utilizar, se listan a continuación junto con la definición operacional que se utilizó dentro de esta investigación.

1. Fácil de usar.

1.1. Facilidad de completar la tarea. Se define como el grado de percepción del usuario en términos de qué tan sencillo le fue completar la tarea asignada.

1.2. Recuperación de errores. Se define como el grado de sencillez en que el usuario pudo continuar con la tarea en caso de errores provocados involuntariamente por él mismo.

1.3. Facilidad para encontrar las acciones a realizar. Se define como el grado mediante el cual el usuario puede interactuar con el sistema para poder ejecutar una tarea. 
1.4. Uso del sistema. Se define como el grado de sencillez de operación percibida por el usuario.

2. Calidad de la Interfaz

2.1. Diseño de la interfaz.

2.1.1. Facilidad de leer el contenido. Se define como el grado en que el usuario puede ver/leer/entender las fuentes utilizadas dentro de la interfaz.

2.1.2. El formato del contenido. Se define como el grado en que la estética del formato le resulta sencillo, agradable y fácil de aprender.

2.1.3. La información desplegada tiene un contenido simple. Se define como el grado de sencillez que tienen tanto los mensajes como la información mostrada para informar al usuario.

2.1.4. Grado de completitud de la información. Se define como el grado en que la información obtenida es suficiente.

2.1.5. La información obtenida es útil. Se define como el grado en que la información proporcionada es considerada como útil.

2.2. Consistencia de las interfaces. Se define como el grado en que la información obtenida es consistente tanto en su presentación como en su ubicación.

2.3. Tiempo necesario.

2.3.1. El tiempo usado para realizar la tarea. Se define como la percepción del usuario acerca del tiempo que le llevó encontrar la tarea que se desea ejecutar.

2.3.2. Tiempo usado para completar la tarea. Se define como la percepción del usuario acerca del tiempo que le llevó completar la tarea desde su inicio hasta su conclusión mediante el sistema, comparada contra realizarla sin utilizar el sistema.

3. Intención de Uso

3.1. Recomendar el sistema a otras personas. Se define como el grado en que un usuario del sistema está dispuesto a recomendar el sistema a otros usuarios.

3.2. Grado de satisfacción con el sistema. Se define como el grado en que un usuario del sistema está satisfecho con el mismo.

3.3. El Grado de completez del Sistema. Se define como la percepción que tiene el usuario de que el sistema realiza todas las tareas identificadas previamente.

Los desarrolladores de sistemas recibieron las especificaciones del proyecto por parte del investigador. Se les dio una semana para estudiarlas y después se llevaron a cabo una serie de reuniones grupales para aclarar 
dudas. Nunca se llevaron a cabo reuniones individuales con el fin de que todos tuvieran la misma información. Una vez aclaradas las dudas, los desarrolladores tuvieron 6 semanas para programar y entregar el sistema. Los sistemas no podían ser ligados al desarrollador. Todos los desarrolladores entregaron el trabajo en forma oportuna.

Los evaluadores de los sistemas desarrollados recibieron una copia de todos los sistemas y tuvieron 3 semanas para evaluarlos. La evaluación consistió en ejecutar el programa y después contestar un cuestionario. El cuestionario está formado por 23 preguntas en una escala de Liekert de 7 puntos acerca de varios aspectos del sistema (la mejor calidad corresponde al 1 y la peor corresponde al 7). Además, los evaluadores podían agregar comentarios de mejoras y errores encontrados. Posteriormente, los resultados de los cuestionarios acerca de su sistema fueron entregados a cada desarrollador con el fin de ver si la calidad está en los valores aceptables. Para efectos del estudio, este proceso se llevó a cabo sólo 2 veces.

\section{ANÁLISIS DE DATOS Grupo con Usabilidad \\ Ronda 1}

La Tabla 2 muestra los datos estadísticos descriptivos de cómo se comportaron los resultados del primer prototipo del SI desarrollado por el GCU. Los resultados muestran que, en promedio, la estética de la interfaz obtuvo la mejor calificación asignada por parte de los evaluadores. Por el contrario, el elemento de ayuda que el sistema debe proveer fue al que se le asignó la calificación más baja.

\begin{tabular}{|c|c|c|c|c|c|c|c|}
\hline & \begin{tabular}{|l} 
Qué tan \\
fácil de \\
usar son \\
las formas \\
que el \\
sistema \\
permite \\
capturar \\
datos. \\
Ronda 1
\end{tabular} & $\begin{array}{l}\text { Qué tan } \\
\text { efectiva- } \\
\text { mente el } \\
\text { sistema } \\
\text { detecta y } \\
\text { maneja } \\
\text { los } \\
\text { errores. } \\
\text { Ronda } 1\end{array}$ & $\begin{array}{l}\text { La ayuda } \\
\text { que el } \\
\text { sistema } \\
\text { proporciona } \\
\text { a los } \\
\text { usuarios } \\
\text { para } \\
\text { ejecutar las } \\
\text { tareas. } \\
\text { Ronda } 1\end{array}$ & $\begin{array}{l}\text { Qué tan } \\
\text { efectiva- } \\
\text { mente el } \\
\text { sistema } \\
\text { valida los } \\
\text { datos } \\
\text { capturados } \\
\text { por los } \\
\text { usuarios. } \\
\text { Ronda } 1\end{array}$ & $\begin{array}{c}\text { Qué tan } \\
\text { atractiva } \\
\text { y estética } \\
\text { son las } \\
\text { interfaces } \\
\text { del } \\
\text { sistema. } \\
\text { Ronda } 1\end{array}$ & \begin{tabular}{|c|} 
Qué tan \\
visible, \\
consistente \\
y estéticos \\
son los \\
tipos de \\
letra \\
usados en \\
el sistema. \\
Ronda 1
\end{tabular} & $\begin{array}{l}\text { Qué tan } \\
\text { efectiva- } \\
\text { mente } \\
\text { son } \\
\text { usados } \\
\text { los } \\
\text { colores } \\
\text { en el } \\
\text { sistema. } \\
\text { Ronda } 1\end{array}$ \\
\hline Válidos & 23 & 23 & 23 & 23 & 23 & 23 & 23 \\
\hline Faltantes & 0 & 0 & 0 & 0 & 0 & 0 & 0 \\
\hline Media & 3.3431 & 3.9704 & 4.8821 & 3.9556 & 3.0540 & 2.5279 & 3.9680 \\
\hline Mediana & 3.2575 & 3.9770 & 5.1800 & 3.9655 & 3.0238 & 2.2419 & 3.9523 \\
\hline Moda & $2.952^{a}$ & 4.613 & 4.065 & 4.151 & 3.602 & $1.910^{\mathrm{a}}$ & 4.667 \\
\hline Desviación Estándar & .6021 & .7437 & 1.1117 & .6575 & .5618 & .7380 & .4715 \\
\hline Factor asimetría & .465 & .611 & -.740 & 1.121 & .204 & 1.606 & .246 \\
\hline Error estd Fact Asim & .481 & .481 & .481 & .481 & .481 & .481 & .481 \\
\hline Curtosis & .01 & .584 & .133 & 2.497 & -.119 & 2.746 & -1.106 \\
\hline Error estd Curtosis & .935 & .935 & .935 & .935 & .935 & .935 & .935 \\
\hline Rango & 2.233 & 3.078 & 4.060 & 3.009 & 2.314 & 3.051 & 1.493 \\
\hline Mínimo & 2.402 & 2.859 & 2.440 & 2.880 & 2.040 & 1.762 & 3.267 \\
\hline Máximo & 4.635 & 5.937 & 6.500 & 5.889 & 4.354 & 4.813 & 4.760 \\
\hline
\end{tabular}

Tabla 2. Estadística descriptiva Ronda 1 (Con Usabilidad) 


\section{Ronda 2}

La Tabla 3 muestra los datos estadísticos descriptivos de cómo se comportaron los resultados del segundo prototipo del SI desarrollado por el GCU, el cual es la versión ya corregida y revisada tomando en cuenta las evaluaciones, errores detectados, sugerencias y opiniones de los evaluadores al primer prototipo. Los resultados muestran que, en promedio, la estética de la interfaz continuó obteniendo la mejor calificación por parte de los evaluadores. Así mismo, el elemento de ayuda continuó obteniendo la calificación más baja por parte de los evaluadores. Esto implica que los usuarios esperan una ayuda más completa y eficiente por parte del sistema. Esto puede indicar que aunque los desarrolladores mejoraron la ayuda del SI, no fue suficiente para los evaluadores.

\begin{tabular}{|c|c|c|c|c|c|c|c|}
\hline & $\begin{array}{l}\text { Qué tan } \\
\text { fácil de usar } \\
\text { son las } \\
\text { formas que } \\
\text { el sistema } \\
\text { permite } \\
\text { capturar } \\
\text { datos. } \\
\text { Ronda } 2\end{array}$ & $\begin{array}{l}\text { Qué tan } \\
\text { efectiva- } \\
\text { mente el } \\
\text { sistema } \\
\text { detecta y } \\
\text { maneja } \\
\text { los } \\
\text { errores. } \\
\text { Ronda } 2\end{array}$ & \begin{tabular}{|c|} 
La ayuda \\
que el \\
sistema \\
proporciona \\
a los \\
usuarios \\
para \\
ejecutar las \\
tareas. \\
Ronda 2
\end{tabular} & \begin{tabular}{|} 
Qué tan \\
efectiva- \\
mente el \\
sistema \\
valida los \\
datos \\
capturados \\
por los \\
usuarios. \\
Ronda 2
\end{tabular} & $\begin{array}{c}\text { Qué tan } \\
\text { atractiva } \\
\text { y estética } \\
\text { son las } \\
\text { interfaces } \\
\text { del } \\
\text { sistema. } \\
\text { Ronda } 2\end{array}$ & $\begin{array}{c}\text { Qué tan } \\
\text { visible, } \\
\text { consistente } \\
\text { y estéticos } \\
\text { son los } \\
\text { tipos de } \\
\text { letra } \\
\text { usados en } \\
\text { el sistema. } \\
\text { Ronda } 2\end{array}$ & 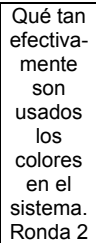 \\
\hline Válidos & 23 & 23 & 23 & 23 & 23 & 23 & 23 \\
\hline Faltantes & 0 & 0 & 0 & 0 & 0 & 0 & 0 \\
\hline Media & 2.1882 & 2.6039 & 3.3856 & 2.8235 & 2.1237 & 1.5644 & 3.1008 \\
\hline Mediana & 2.0392 & 2.4791 & 3.4347 & 2.7017 & 2.1428 & 1.5625 & 3.3157 \\
\hline Moda & $1.439^{a}$ & $1.854^{\mathrm{a}}$ & $1.652^{a}$ & 3.794 & $1.574^{a}$ & 1.452 & $1.981^{a}$ \\
\hline Desviación Estándar & .4627 & .5303 & 1.0628 & .5703 & .3813 & .1856 & .5299 \\
\hline Factor asimetría & 1.156 & 1.671 & .142 & 1.365 & 1.018 & .675 & -1.064 \\
\hline Error estd Fact Asimetría & .481 & .481 & .481 & .481 & .481 & .481 & .481 \\
\hline Curtosis & 1.487 & 3.074 & -.622 & 2.269 & 2.704 & 1.254 & .065 \\
\hline Error estd de Curtosis & .935 & .935 & .935 & .935 & .935 & .935 & .935 \\
\hline Rango & 2.022 & 2.289 & 3.693 & 2.416 & 1.712 & .839 & 1.892 \\
\hline Mínimo & 1.439 & 1.854 & 1.652 & 2.061 & 1.574 & 1.224 & 1.981 \\
\hline Máximo & 3.460 & 4.143 & 5.345 & 4.476 & 3.286 & 2.063 & 3.873 \\
\hline
\end{tabular}

Tabla 3. Estadística descriptiva Ronda 2 (Con Usabilidad)

Ahora bien, al comparar los resultados de ambas rondas podemos observar lo siguiente:

- Como ya se mencionó previamente, la mejor calidad es aquella que obtiene un valor de 1 o cercano a él, y la peor es la que obtiene el valor de 7 o cercano a él. La media se reduce en cada uno de los siete componentes evaluados de forma importante, es decir, permite ver que la calidad se mejora del primer al segundo prototipo notablemente.

- En seis de los siete componentes la desviación estándar se reduce en la segunda ronda comparada con la primera ronda, lo cual quiere decir que los evaluadores asignaron valores menos dispersos. Esto puede comprobarse al ver el valor mínimo y máximo asignados durante las evaluaciones. 
Adicionalmente, los resultados de las dos rondas fueron comparados utilizando pruebas $t$, cuyos resultados se pueden ver en la Tabla 4. Es muy importante remarcar que el grupo con usabilidad muestra que en todos los componentes se incrementó la calidad relativa obteniendo una muy alta significancia $(\mathrm{p}<.001)$. Entonces, utilizar la teoría de usabilidad realmente hace la diferencia al desarrollar y probar sistemas de software.

Tabla 4. Resultados de pruebas t apareadas (Con Usabilidad)

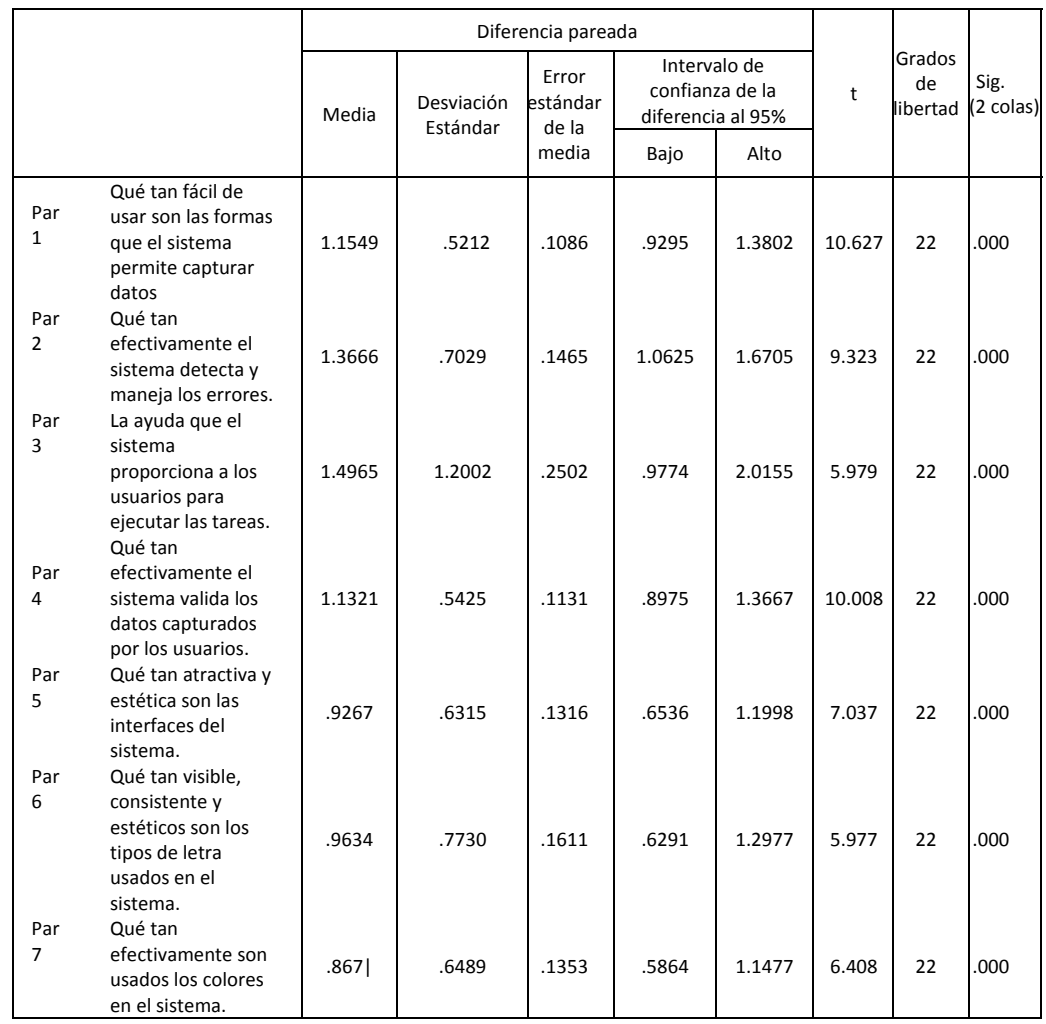

\section{Grupo Sin Usabilidad Ronda Única}

La Tabla 5 muestra los datos estadísticos descriptivos de cómo se comportaron los resultados en la ronda 1 con usabilidad. Analizándola se puede observar lo siguiente:

Considerando que el mejor valor de calidad es 1 se puede notar que en todos los elementos la calidad es insuficiente. El mejor valor obtenido es 2.57 en aspectos de visibilidad estética, pero no podría considerarse como suficiente. El resto de los elementos pueden ser considerados como de calidad mediocre ya que están muy cerca de la media natural (facilidad de 
uso de formas y estética de la interfaz) del la evaluación (3.5 punto) o están más allá de la mediocridad (manejo de errores, ayuda, validación y uso de color). En consecuencia, el SI final requiere una mejora sustancial en los elementos de calidad evaluados ya que de no hacerlo el usuario podría rechazar la adopción del sistema, o causar pérdidas en recursos (tiempo, materiales, etc.) e incluso económicas. Es importante pues que se lleve a cabo una serie de acciones que mejoren sustancialmente la calidad final del SI entregado para su implantación.

\begin{tabular}{|c|c|c|c|c|c|c|c|}
\hline & $\begin{array}{l}\text { Qué tan } \\
\text { fácil de } \\
\text { usar son } \\
\text { las formas } \\
\text { que el } \\
\text { sistema } \\
\text { permite } \\
\text { capturar } \\
\text { datos. }\end{array}$ & $\begin{array}{l}\text { Qué tan } \\
\text { efectiva- } \\
\text { mente el } \\
\text { sistema } \\
\text { detecta y } \\
\text { maneja los } \\
\text { errores. }\end{array}$ & $\begin{array}{c}\text { La ayuda } \\
\text { que el } \\
\text { sistema } \\
\text { proporciona } \\
\text { a los } \\
\text { usuarios } \\
\text { para } \\
\text { ejecutar las } \\
\text { tareas. }\end{array}$ & $\begin{array}{l}\text { Qué tan } \\
\text { efectiva- } \\
\text { mente el } \\
\text { sistema } \\
\text { valida los } \\
\text { datos } \\
\text { capturados } \\
\text { por los } \\
\text { usuarios }\end{array}$ & $\begin{array}{c}\text { Qué tan } \\
\text { atractiva } \\
\text { y estética } \\
\text { son las } \\
\text { interfaces } \\
\text { del } \\
\text { sistema. }\end{array}$ & $\begin{array}{l}\text { Qué tan } \\
\text { visible, } \\
\text { consistente } \\
\text { y estéticos } \\
\text { son los } \\
\text { tipos de } \\
\text { letra } \\
\text { usados en } \\
\text { el sistema. }\end{array}$ & $\begin{array}{l}\text { Qué tan } \\
\text { efectiva- } \\
\text { mente } \\
\text { son } \\
\text { usados } \\
\text { los } \\
\text { colores } \\
\text { en el } \\
\text { sistema. }\end{array}$ \\
\hline Válidos & 20 & 20 & 20 & 20 & 20 & 20 & 20 \\
\hline Faltantes & 0 & 0 & 0 & 0 & 0 & 0 & 0 \\
\hline Media & 3.2484 & 3.8092 & 4.6886 & 3.7957 & 3.0429 & 2.5752 & 3.9059 \\
\hline Mediana & 3.2455 & 3.9220 & 4.9779 & 3.8935 & 3.0536 & 2.3390 & 3.7795 \\
\hline Moda & 2.914 & $2.827^{a}$ & $2.148^{a}$ & $2.950^{\mathrm{a}}$ & $2.040^{a}$ & $1.809^{a}$ & 4.751 \\
\hline Desv Estándar & .5573 & .5996 & 1.1423 & .4955 & .5407 & .7520 & .4938 \\
\hline Factor asimetría & .274 & -.141 & -.734 & .087 & .068 & 1.483 & .506 \\
\hline Err estd Fact Asim & .612 & .512 & .512 & .512 & .512 & .512 & .512 \\
\hline Curtosis & -.121 & -1.321 & .111 & .652 & .181 & 2.549 & -.827 \\
\hline Error estd Curtosis & .992 & .992 & .992 & .992 & .992 & .992 & .992 \\
\hline Rango & 2.161 & 1.754 & 4.187 & 2.035 & 2.214 & 2.980 & 1.563 \\
\hline Mínimo & 2.350 & 2.847 & 2.148 & 2.950 & 2.040 & 1.809 & 3.188 \\
\hline Máximo & 4.511 & 4.601 & 6.335 & 4.985 & 4.254 & 4.789 & 4.751 \\
\hline
\end{tabular}

Tabla 5. Estadística descriptiva Ronda 2 (Sin Usabilidad)

\section{Comparación de Ambos Grupos}

Con el fin de verificar si existen diferencias en los resultados de calidad entre ambos estudios realizados se llevaron a cabo pruebas t. Esto permitió comparar los resultados finales en el efecto de la participación de los usuarios en la evaluación de la calidad de los SI en ambos estudios. Los resultados de dichas pruebas se muestran en la Tabla 6. Podemos observar que en todos los aspectos los resultados son mejores usando Usabilidad (estudio donde participaron activamente los usuarios) comparado con Nousabilidad (solo participaron al final del estudio). En consecuencia, esto permite confirmar la hipótesis de la investigación de que la participación de los usuarios en la evaluación de la calidad de los SI ayuda a obtener mejores productos de software $(\mathrm{p}<.01)$. 


\begin{tabular}{|c|c|c|c|c|c|c|c|c|c|c|}
\hline & \multicolumn{2}{|c|}{$\begin{array}{l}\text { Prueba de Levene para } \\
\text { Varianzas Equivalentes }\end{array}$} & \multicolumn{7}{|c|}{ Prueba t para Medias Equivalentes } \\
\hline & & \multirow[b]{2}{*}{$\mathrm{F}$} & \multirow[b]{2}{*}{ sig. } & \multirow[b]{2}{*}{$t$} & \multirow[b]{2}{*}{$d f$} & \multirow{2}{*}{$\begin{array}{l}\text { Sinnificancia } \\
\text { (2 colas) }\end{array}$} & \multirow{2}{*}{$\begin{array}{l}\text { Diferencia de } \\
\text { Medias }\end{array}$} & \multirow{2}{*}{$\begin{array}{l}\text { Diferencia } \\
\text { Error estándar }\end{array}$} & \multicolumn{2}{|c|}{$\begin{array}{l}\text { Intervalo de confianza } \\
\text { de la Diferencia al } 95 \%\end{array}$} \\
\hline & & & & & & & & & Bajo & Alto \\
\hline $\begin{array}{l}\text { Diferencia entre fácil de } \\
\text { usar en Ronala } 1 \text { compa- } \\
\text { rada can Ronda } 2\end{array}$ & $\begin{array}{l}\text { Se asumen } \\
\text { Varlanzas iguales } \\
\text { No se asumen } \\
\text { varianzas iguales }\end{array}$ & 22.757 & .000 & $\begin{array}{r}9.464 \\
10.145\end{array}$ & $\begin{array}{r}41 \\
22.712\end{array}$ & $\begin{array}{l}.000 \\
.000\end{array}$ & $\begin{array}{r}1.111410 \\
1.111410\end{array}$ & $\begin{array}{l}.117440 \\
.109558\end{array}$ & $\begin{array}{l}.874234 \\
.884613\end{array}$ & $\begin{array}{l}1.348585 \\
1.338206\end{array}$ \\
\hline $\begin{array}{l}\text { Diferencia entre manejo de } \\
\text { error Ronda } 1 \text { comparado } \\
\text { con Ronda } 2\end{array}$ & $\begin{array}{l}\text { Se asumen } \\
\text { Varianzas iguales } \\
\text { No se asumen } \\
\text { varianzas iguales }\end{array}$ & 36.552 & .000 & $\begin{array}{l}8.371 \\
8.986\end{array}$ & $\begin{array}{r}41 \\
22.197\end{array}$ & $\begin{array}{l}.000 \\
.000\end{array}$ & $\begin{array}{l}1.32008 .2 \\
1.32008 .2\end{array}$ & $\begin{array}{l}.157706 \\
.146910\end{array}$ & $\begin{array}{l}1.001588 \\
1.015566\end{array}$ & $\begin{array}{l}1.638576 \\
1.624598\end{array}$ \\
\hline $\begin{array}{l}\text { Diferencia entre ayuda pro- } \\
\text { porcianada en Ronda } 1 \\
\text { comparada con Ronda } 2\end{array}$ & $\begin{array}{l}\text { Se asumen } \\
\text { Varianzas iguales } \\
\text { No sc asumen } \\
\text { varianzas iguales }\end{array}$ & 31.328 & .000 & $\begin{array}{l}5.360 \\
5.749\end{array}$ & $\begin{array}{r}41 \\
22.495\end{array}$ & $\begin{array}{l}.000 \\
.000\end{array}$ & $\begin{array}{l}1.446857 \\
1.446857\end{array}$ & $\begin{array}{l}.269950 \\
.251679\end{array}$ & $\begin{array}{l}.901682 \\
.925571\end{array}$ & $\begin{array}{l}1.992032 \\
1.968143\end{array}$ \\
\hline $\begin{array}{l}\text { Diferencia entre validación } \\
\text { de datos en Ronda } 1 \\
\text { comparada con Ronda } 2\end{array}$ & $\begin{array}{l}\text { Se asumen } \\
\text { Varianzas iguales } \\
\text { No se asumen } \\
\text { varianzae igualee }\end{array}$ & 10.364 & .000 & $\begin{array}{l}8.920 \\
9.551\end{array}$ & $\begin{array}{r}41 \\
23.109\end{array}$ & $\begin{array}{l}.000 \\
.000\end{array}$ & $\begin{array}{l}1.094014 \\
1.094014\end{array}$ & $\begin{array}{l}.122651 \\
.114543\end{array}$ & $\begin{array}{l}.846315 \\
.857125\end{array}$ & $\begin{array}{l}1.341713 \\
1.330903\end{array}$ \\
\hline $\begin{array}{l}\text { Diferencia entre diseño de } \\
\text { interfaces en Ronda } 1 \\
\text { comparada con Ronda } 2 \text {. }\end{array}$ & $\begin{array}{l}\text { Se asumen } \\
\text { Varianzas iguales } \\
\text { No seasumen } \\
\text { varianzas iguales }\end{array}$ & 21.079 & .000 & $\begin{array}{l}6.380 \\
6.828\end{array}$ & $\begin{array}{r}41 \\
23.307\end{array}$ & $\begin{array}{l}.000 \\
.000\end{array}$ & $\begin{array}{l}.912477 \\
.912477\end{array}$ & $\begin{array}{l}.143028 \\
.133645\end{array}$ & $\begin{array}{l}.623627 \\
.636213\end{array}$ & $\begin{array}{l}\text { 1. } 201327 \\
\text { 1. } 188741\end{array}$ \\
\hline $\begin{array}{l}\text { Diferencia entre tipas de } \\
\text { fuentes usados en Ronda } 1 \\
\text { comparada con Ronda } 2 \text { ? }\end{array}$ & $\begin{array}{l}\text { Se asumen } \\
\text { Varianzas iguales } \\
\text { No se asumen } \\
\text { varianzas iguales }\end{array}$ & 17.454 & .000 & $\begin{array}{l}5.292 \\
5.673\end{array}$ & $\begin{array}{r}41 \\
22.674\end{array}$ & $\begin{array}{l}.000 \\
.000\end{array}$ & $\begin{array}{l}.921435 \\
.921435\end{array}$ & $\begin{array}{l}.17412 a \\
.162416\end{array}$ & $\begin{array}{l}.569792 \\
.505104\end{array}$ & $\begin{array}{l}\text { 1. } 273077 \\
\text { 1. } 257605\end{array}$ \\
\hline $\begin{array}{l}\text { Diferencia entre usa de } \\
\text { calores en Ronda } 1 \\
\text { camparado can Randa } 2\end{array}$ & $\begin{array}{l}\text { Se asumen } \\
\text { Varianzas iguales } \\
\text { No se asumen } \\
\text { varianzas iguales }\end{array}$ & 45.261 & .000 & 5.38 .3 & 24.129 & $\begin{array}{l}.000 \\
.000\end{array}$ & .796552 & .147971 & .497719 & 1. 095385 \\
\hline
\end{tabular}

Tabla 6. Resultados de pruebas t comparando Usabilidad vs. No-usabilidad

\section{CONCLUSIONES}

Todo proyecto trata de maximizar sus esfuerzos y recursos. El desarrollo de software no es diferente. Entonces, el crear un nuevo producto siempre debe ser teniendo esta principio en mente. En el estudio presentado se encontraron resultados alentadores que podrían coadyuvar en lograr la optimización en desarrollos de software. Podemos notar que los resultados muestran que el uso de un método estructurado en el desarrollo de software evaluándolo por usabilidad, en el cual se utilice la estadística como herramienta, permite incrementar la calidad de los SI. Como une primera aproximación se evaluaron siete elementos y todos ellos fueron mejorados -como las pruebas lo muestran. Esto podría representas una mejor forma de evaluación del software, ya que se incrementa la calidad de los sistemas en un tiempo corto, lo cual a su vez representa una reducción de costos para la empresa.

Al hacer múltiples búsquedas en varias bases de datos de revistas electrónicas nos dimos cuenta que las teorías de usabilidad no han sido muy investigadas y/o utilizadas en México, especialmente en la región donde se llevó a cabo la investigación, ya que prácticamente no se encontró artículo alguno que haga referencia a dicho componente de los SI. Dichas teorías están basadas en las pruebas empíricas realizadas. Entonces, los participantes podrán aprender una forma diferente de evaluación de la calidad, especialmente a SI. Adicionalmente, esta investigación puede contribuir a que los investigadores participantes transmitan dichos conocimientos a sus estudiantes en materias que tienen que ver con desarrollo y mantenimiento de SI.

Los SI son un elemento muy importante para que las organizaciones permanezcan competitivas. Más aún, a través de los SI es como la vasta mayoría de ellas lleva a cabo un gran número de sus actividades diarias. En consecuencia, es de vital importancia que los SI realmente sirvan para que el usuario final pueda cumplir con sus tareas y contribuya al 
funcionamiento de la organización. Un sistema debe ser desarrollado tomando en cuenta los aspectos que los usuarios finales consideran como primordiales, así como minimizar la posibilidad de errores de diseño y/o funcionamiento. De lo contrario, se puede incrementar la resistencia al cambio natural de los usuarios hacia el nuevo SI. Creemos que al identificar una forma en la que los usuarios se involucran directamente con el desarrollo del software así como con la evaluación del mismo, puede coadyuvar a que los desarrolladores de SI generen productos más eficientes y de mayor calidad, reduciendo con esto el costo más grande de todo software: el mantenimiento.

\section{LIMITACIONES Y TRABAJOS FUTUROS}

El presente estudio arroja resultados alentadores. Sin embargo, estos resultados deben tomarse con cautela debido a la forma en que éste se condujo. En las organizaciones el medio ambiente de trabajo es diferente y existen presiones en los usuarios que tienen relación con el uso de SI. Una limitante es el hecho que los evaluadores fueron estudiantes de licenciatura, lo cual podría no representar al usuario final del sistema. La gran mayoría de los estudiantes que participaron como evaluadores trabajan pero el SI evaluado no forma parte de su trabajo, es decir, lo hicieron como un trabajo extracurricular. En consecuencia, podrían haber ignorado -consciente o inconscientemente- aspectos que no harían en un SI para el desempeño de su trabajo. Entonces, un estudio en el cual participen usuarios finales reales podría producir resultados diferentes así como más información acerca del proceso de desarrollo de SI.

Adicionalmente, solo se evaluaron siete elementos de un SI. Entonces, los resultados obtenidos podrían ser verdaderos para estos elementos por lo que un estudio que incremente el número de elementos podría arrojar resultados diferentes.

En la actualidad se está ampliando el presente estudio implicando más elementos en la evaluación de SI así como conducida con usuarios reales que trabajan en dos organizaciones; una pública y una privada.

\section{LECTURAS ADICIONALES RECOMENDADAS}

Dennis, A., Wixom, B. H., \& Roth, R. M. (2006). Systems Analysis and Design. Honoken, NJ: John Wiley and Sons.

Dennis, A., Wixom, B. H., \& Tegarden, D. (2002). Systems Analysis and Design. An Object-Oriented Approach with UML. New York: John Wiley and Sons.

Dumas, J. F., \& Redish, J. C. (1999). A Practical Guide to Usability Testing. Bristol, England: Intellect Books.

ISO. (1991). Information Technology - Software Product Evaluation Quality Characteristics and Guidelines for Their Use. In ISO (Ed.) (Vol. ISO 9126). 
McCall, J., Richards, P., \& Walters, G. (1977). Factors in Software Quality. Volumes I, II and III: Rome Air Development Center ReportsNTIS AD/A-049 014, NTIS AD/A-049 015 and NTIS AD/A049 016, U. S. Department of Commerce.

Pressman, R. S. (2005). Software Engineering: A Practitioner's Approach, 6/e. New York, N.Y.: Mc Graw-Hill.

Satzinger, J. W., Jackson, R. B., \& Burd, S. D. (2009). Systems analysis and design in a changing world. Boston, MA: Thomson Learning.

Sommerville, I. (2006). Software Engineering. 8/e. London, U.K.,: Addison-Wesley.

Turban, E., Leidner, D., McLean, E., \& Wetherbe, J. (2006). Information Technology for Management: Transforming Organizations in the Digital Economy.

Turban, E., McLean, E., \& Wetherbe, J. (2001). Information Technology for Management. Making Connections for Strategic Advantage. 2nd. edition. New York: John Wiley \& Sons.

\section{BIBLIOGRAFIA}

Amasaki, S., Yoshitomi, T., Mizuno, O., Takagi, Y., \& Kikuno, T. (2005). A New Challenge for Applying Time Series Metrics Data to Software Quality Estimation. Software Quality Journal, 13(2), 177-193.

Applegate, L. M., Austin, R. D., \& McFarlan, F. W. (2003). Corporate Information Strategy abd Management, 6th edition (6th ed.). New York, N.Y.: Mc. Graw-Hill.

Boehm, B. (2006). A View of 21st Century Software Engineering. Paper presented at the ICSE'06, Shangai, China.

Bosch, J., \& Juristo, N. (2003). Designing Software Architectures for Usability. Paper presented at the 25th International Conference on Software Engineering, Portland, Oregon.

Buch, K., \& Bartley, S. (2002). Learning Style and Training Delivery Mode Preference. Journal of Workplace Learning, 014(1), 5-10.

Constantine, L. L., \& Dockwood, L. A. D. (2003). Usage-Centered Software Engineering: An Agile Approach to Integrating Users, User Interfaces, and Usability into Software Engineering Practice. Paper presented at the 25th International Conference on Software Engineering, Portland, Oregon, USA.

De Villiers, R. (2004). Usability evaluation of an E-Learning tutorial: Criteria, questions and case study. Paper presented at the SAICSIT 2004, Stellenbosch, Western Cape, South Africa.

Dennis, A., Wixom, B. H., \& Roth, R. M. (2006). Systems Analysis and Design. Honoken, NJ: John Wiley and Sons.

Dennis, A., Wixom, B. H., \& Tegarden, D. (2002). Systems Analysis and Design. An Object-Oriented Approach with UML. New York: John Wiley and Sons.

Drucker, P. F. (1993). The Effective Executive. Harper Business Press, New York. 
Drucker, P. F. (1999). Management Challenges for the 21st Century. Harper Business Press, New York.

Dumas, J. F., \& Redish, J. C. (1999). A Practical Guide to Usability Testing. Bristol, England: Intellect Books.

ISO. (1991). Information Technology - Software Product Evaluation Quality Characteristics and Guidelines for Their Use. In ISO (Ed.) (Vol. ISO 9126).

Juristo, N., \& Ferre, X. (2006). How to Integrate Usability into the Software Development Process. Paper presented at the 28th international conference on Software engineering, Shanghai, China.

Kekäle, T., Pirolt, K., \& Falter, C. (2002). IT Tools in Personnel Training in Small Enterprises: Results of the Project "Apply". Journal of Workplace Learning, 14(7), 269-277.

McCall, J., Richards, P., \& Walters, G. (1977). Factors in Software Quality. Volumes I, II and III: Rome Air Development Center ReportsNTIS AD/A-049 014, NTIS AD/A-049 015 and NTIS AD/A049 016, U. S. Department of Commerce.

Mehlenbacher, B. (1993). Software usability: Choosing appropriate methods for evaluating online systems and documentation. Paper presented at the SIGDOC '93, Waterloo, Ontario, Canada.

Miller, D. M. (1995). Productivity Through People. Employee Counseling Today, 7(4), 1-19.

Nunamaker, J. F. (1992). Build to Learn, Evaluate and Learn. Informatica $1,1,1-6$.

Pressman, R. S. (2005). Software Engineering: A Practitioner's Approach, 6/e. New York, N.Y.: Mc Graw-Hill.

Rutherford, M. A., \& Ramey, J. A. (2000). The design response to usability test findings: A case study based on artifacts and interviews. Paper presented at the 18th annual ACM international conference on Computer documentation: technology \& teamwork, Cambridge, Massachusetts.

Satzinger, J. W., Jackson, R. B., \& Burd, S. D. (2009). Systems analysis and design in a changing world. Boston, MA: Thomson Learning.

Sommerville, I. (2006). Software Engineering. 8/e. London, U.K.,: Addison-Wesley.

Thompson, S. M. (2003). Remote Observation Strategies for Usability Testing. Information Technology and Libraries, 22(1), 22.

Tiwana, A., \& McLean, E. R. (2005). Expertise Integration in Information Systems Development. Journal of Management Information Systems, 22(1), 13-43.

Turban, E. (1995). Decision Support and Expert Systems Management Support Systems fourth edition. Prentice Hall.

Turban, E., Leidner, D., McLean, E., \& Wetherbe, J. (2006). Information Technology for Management: Transforming Organizations in the Digital Economy. 
Turban, E., McLean, E., \& Wetherbe, J. (2001). Information Technology for Management. Making Connections for Strategic Advantage. 2nd. edition. New York: John Wiley \& Sons.

UW. (2004, October 05, 2004). Usability test for the University of Washington libraries' information gateway. Retrieved December 2004, 2005 ,

from http://www.lib.washington.edu/usability/redesign0304/surveyResults.html

Walls, J. G., Widmeyer, G. R., \& El Sawy, O. A. (1992). Building an Information System Design Theory for Vigilant EIS. Information Systems Research, 3, 36-58.

Walls, J. G., Widmeyer, G. R., \& El Sawy, O. A. (2004). Assessing Information System Design Theory in Perspective: How Useful was Our 1992 Initial Rendition? Journal of Information Technology Theory and Application, 6(2), 43-58.

Zayaraz, G., Thambidurai, P., Srinivasan, M., \& Rodrigues, P. (2005). Software quality assurance through COSMIC FFP. ACM SIGSOFT Software Engineering Notes, 30(5), 5 pages.

Juan Manuel Gómez Reynoso es Ingeniero en Sistemas Computacionales y estudió la maestría en Informática y Tecnologías Computacionales y la Especialidad en Redes en la Universidad Autónoma de Aguascalientes (UAA), México. Posteriormente, estudió la maestría en Ciencias en Sistemas de Información y el Doctorado en Ciencias en Sistemas de Información en Claremont Graduate University, USA. Es profesor de tiempo completo de la UAA desde 1990. Actualmente es coordinador de la carrera de Ing. en Sistemas Computacionales y de la Maestría en Redes de Computadoras. Ha publicado artículos en revistas internacionales y nacionales, así como participado en congresos internacionales tales como AMCIS, IIMA, Conf-IRM, SDISC, entre otros. Sus principales intereses de investigación son: Desarrollo de software mediante Teorías de Diseño de Sistemas de Información (ISDT), medición de la calidad de software, cómputo móvil, y participación de los usuarios en la evaluación de sistemas.

Mónica Brizuela Sandoval es Ingeniera en Sistemas Computacionales por la Universidad Autónoma de Aguascalientes (UAA), México. Se ha desempeñado como profesor de asignatura de la UAA desde el año 2000. En ese mismo año, comenzó a participar en investigaciones relacionadas al desarrollo de software con calidad y ha sido coautora de artículos en revistas internacionales y nacionales. Sus principales intereses de investigación son: medición de la calidad de software y participación de los usuarios en la evaluación de sistemas. 


\section{Política Editorial}

RELCASI está principalmente dirigida a investigadores de habla hispana en el área de Administración de Sistemas de Información (MIS por sus siglas en Inglés). Los artículos son académicamente rigurosos sin sacrificar la claridad, estilo, simplicidad y contribución práctica que los hace atractivos a profesionales de la disciplina. En consecuencia, la audiencia de esta revista está compuesta no sólo por investigadores de MIS, sino también por profesionales y administradores en el área de tecnologías de información.

Todos los artículos son escritos, revisados y publicados en español; sin embargo, estos contendrán título, resumen y palabras claves en español y en inglés.

RELCASI es una publicación arbitrada que se presenta en formato impreso y en línea. La versión impresa de RELCASI está disponible a pedido (y próximamente bajo subscripción). La versión en línea se provee a través de la Asociación de Sistemas de Información. El proceso de evaluación se realizará con al menos dos examinadores. La identidad del editor asociado y de los examinadores no será del conocimiento del autor, y ni los examinadores ni el editor asociado conocerán la identidad del autor. Una ronda del proceso de evaluación tomará alrededor de 90 días.

La revista incluye principalmente artículos de investigación desarrollados con un marco teórico robusto y que incluyan una adecuada revisión de literatura. Los artículos podrán ser de investigación empírica (cualitativa o cuantitativa), conceptuales, encuestas de corrientes de investigación, o encuestas de la industria de TI en países en desarrollo. Los artículos de investigación empírica, deben proveer una amplia justificación y descripción de la colección de datos, metodología y técnicas analíticas. Estudios de caso, artículos pedagógicos, revisión de libros, y debates y ensayos de opinión serán considerados pero no formarán el grueso de la publicación. Artículos con un alto contenido técnico y bajo contenido gerencial/administrativo no son recomendados y sólo serán aceptados cuando sean altamente relevantes o innovadores. Los artículos deberán tener una extensión no mayor a las 8.000 palabras. 


\section{Editorial Policy}

RELCASI is primarily directed to Spanish speaking researchers in the area of Management Information Systems (MIS). Articles will have academic rigor without sacrificing clarity, style, simplicity, and a practical contribution that will also make them attractive to practitioners. Therefore, its audience includes both academics and practitioners of MIS and IT.

Articles are written, reviewed, and published in Spanish; however, their title, abstract, and keywords will also be published in English.

RELCASI is a double-blind peer-reviewed journal that is both in-print and on-line. The print version is currently provided on-demand and we will soon have a subscription service. The on-line version is available through the Association for Information Systems. The double-blind peer-review process will involve an associate editor and a minimum of two academic peers. We aim to have a round of the review process take no more than 90 days.

The journal will primarily comprise of research articles developed with a robust theoretical framework that include an appropriate literature review. The articles could be qualitative or quantitative, conceptual, research stream surveys, or surveys that relate to IT/MIS in developing countries. Empirical research articles must include a clear, comprehensive, and concise description of the methodology, data collection, and analytical techniques used. Case studies, pedagogical articles, book reviews, debates, and opinion papers will be considered but will not make the bulk of the journal. Articles with a high technical and low managerial content are not encouraged but may be accepted if highly relevant or innovative. Articles may not include more than 8,000 words. 


\section{Solicitud de Artículos \\ Call for Articles}

RELCASI está permanentemente en la búsqueda de artículos en español en el área de sistemas de información (MIS), la cual incluye tópicos relacionados a la adopción, administración, uso, e impactos de la tecnología de información (TI). Tópicos populares incluyen (pero no están limitados a) los siguientes:

- Estudios inter-culturales (dentro de países latinoamericanos o comparaciones con países desarrollados) que comparen antecedentes e impactos de la TI en organizaciones

- Modelos de bases de datos y estructuras de sistemas de información a nivel empresarial o global

- Factores culturales que influyen en el desarrollo efectivo de sistemas de información a gran escala

- Costos y Retornos de Inversión esperados en la implementación de tecnologías de información

- Impacto de TI emergente en pequeñas y medianas empresas (PYMEs)

- El rol de TI en mejorar la ventaja competitiva de las PYMEs

- Infraestructura de sistemas de información

- Recursos humanos en sistemas de información

- Impacto de la TI en la productividad individual

- Programas personalizados vs encapsulados

- Efectos de obligar el uso de TI específicas en subsidiarias locales

- Tercerización ("outsourcing") / Offshoring / Nearshoring

- Debates acerca de implementaciones globales

- Procura de TI en países en vías de desarrollo

- Uso, difusión y legislación de programas de código abierto en Latinoamérica

- Costo total: programas, adaptación, consultoría y entrenamiento

- Como programas de código abierto pueden contribuir al desarrollo

- Uso, venta, e implementación de paquetes globales en economías locales

- Impacto de estilos gerenciales en el uso y desarrollo de TI

- Ejecución de contratos de TI en una economía global

- La paradoja de la productividad de la TI en Latinoamérica

- Implementación y adaptación de paquetes de programas

- Implementaciones globales

- Transferencia de tecnología

- Aspectos económicos y financieros de la compra, desarrollo e implementación de TI 
- Debates en sistemas de entrenamiento de TI (para expertos y usuarios)

- El valor del negocio de la TI

- Soporte local vs soporte a larga distancia

Los artículos pueden utilizar cualquiera de las siguientes modalidades de acuerdo al contexto y metodología.

1. Investigación empírica

a. Cualitativa (perspectivas positivistas o interpretativas): desarrollo o comprobación de teorías: estudios de caso, estudios de casos múltiples, investigación-acción

b. Cuantitativa: comprobación de teorías: experimentos, encuestas, estudios de caso, archivos

2. Encuestas de corrientes de investigación: revisión de literatura usando narrativa o meta-análisis

3. Encuestas de la industria de TI en países en desarrollo. Debido a la falta de difusión del conocimiento de TI en países en desarrollo es importante publicar artículos que provean una visión general de la situación de la industria de TI en estos países. Los artículos pueden ser reportes académicos que provean una clara representación de la industria de TI y/o su relación con otras industrias y el gobierno.

4. Conceptual: desarrollo de nuevas teorías/modelos desde literatura existente, observación de hechos y argumentos lógicos

5. Diseño de la investigación: desarrollo de artefactos para resolver problemas relevantes que profesionales de la TI enfrentan en países en desarrollo. Algunos ejemplos pueden incluir: desarrollo de herramientas y aplicaciones innovadoras de TI, nuevos métodos para gerenciar TI en países en desarrollo, etc.

Artículos con un alto contenido técnico y bajo contenido gerencial/administrativo no son recomendados y solo serán aceptados cuando sean altamente relevantes o innovadores. La revista incluirá principalmente artículos teóricos $\mathrm{y}$ de investigación que han sido desarrollados en un marco teorético robusto, incluyen una adecuada revisión de literatura y proveen una amplia justificación y descripción de la metodología y técnicas analíticas. Estudios de caso, artículos pedagógicos, revisión de libros, y debates y ensayos de opinión serán considerados pero no formarán parte del grueso de la publicación. 


\section{AUDIENCIA}

La revista está principalmente dirigida a investigadores de MIS de habla hispana. Los artículos serán académicamente rigurosos sin sacrificar la claridad, estilo y simplicidad que hace que estos artículos sean atractivos a profesionales de la disciplina En consecuencia, la revista será atractiva no solo para investigadores de MIS y sino también para profesionales.

\section{IDIOMA}

Todos los artículos serán escritos, revisados y publicados en español; sin embargo, el título, palabras claves y resumen deberán ser incluidos en inglés y español

\section{FORMATO}

La revista es una publicación arbitrada que se presentará en formato impreso y en línea. La versión impresa de la revista estará disponible a pedido. La versión electrónica será publicada en el sitio de AIS. Los artículos deberán tener una extensión no mayor a las 8.000 palabras.

El proceso de evaluación se realizara con al menos dos árbitros. La identidad del editor asociado y de los examinadores no será del conocimiento del autor y estos tampoco conocerán la identidad del autor. Una ronda del proceso de evaluación deberá tomar alrededor de 90 días.

Los artículos deben ser enviados directamente a través del sistema automatizado en http://mc.manuscriptcentral.com/relcasi Para preguntas y sugerencias envíe un correo electrónico a editor@relcasi.org Información adicional está disponible en www.relcasi.org 